\title{
Standardization of Prepackaging Materials and Storage Environment on Post Harvest Shelf Life of Bitter Gourd (Momordica charantia L.)
}

\author{
Rajasekar Manivelu $^{1 *}$, Swaminathan Venkatesan ${ }^{2}$ and Hemalatha Ganapathyswami $^{3}$ \\ ${ }^{1}$ Precision Farming Development Centre, Agricultural Engineering College and Research \\ Institute, Tamil Nadu Agricultural University, Coimbatore - 641 003, Tamil Nadu, India \\ ${ }^{2}$ Horticultural College and Research Institute, ${ }^{3}$ Home Science College and Research Institute, \\ Tamil Nadu Agricultural University, Madurai - 625 104, Tamil Nadu, India \\ *Corresponding author
}

\begin{tabular}{|c|c|}
\hline & A B S T R A C T \\
\hline Keywords & $\begin{array}{l}\text { An investigation was carried out to study the effect of prepackaging materials on } \\
\text { post harvest shelf life of Bitter gourd cultivars } C_{1}-\text { Roma, } C_{2}-C O 1, C_{3^{-}} \text {MAHY }\end{array}$ \\
\hline $\begin{array}{l}\text { Bitter gourd, } \\
\text { Cultivars, } \\
\text { Packaging, } \\
\text { Shelf life, } \\
\text { Storage }\end{array}$ & $\begin{array}{l}101, \mathrm{C}_{4^{-}} \text {US } 6214 \text { and } \mathrm{C}_{5^{-}} \text {Palee. There were six treatments in factor one Viz., } \mathrm{T}_{1-} \\
\text { Perforated poly bag }\left(200 \text { gauge }+1 \% \text { ventilation), } \mathrm{T}_{2} \text {-Unperforated poly bag ( } 200\right. \\
\text { gauge), } \mathrm{T}_{3}-\text { Wetted gunny bag, } \mathrm{T}_{4^{-}} \text {Wetted cloth bag, } \mathrm{T}_{5^{-}} \text {CFB box }(1 \% \\
\text { ventilation) and } \mathrm{T}_{6^{-}} \text {Control (without any packing) and two treatments in factor }\end{array}$ \\
\hline Article Info & $\begin{array}{l}\text { two Viz., Retrigerated condition }\left(S_{1}\right) \text { and Ambient condition }\left(S_{2}\right) \text {. The results } \\
\text { revealed that, the treatment } T_{1} \text { - Perforated poly bag }(200 \text { gauge }+1 \% \text { ventilation })\end{array}$ \\
\hline $\begin{array}{l}\text { Accepted: } \\
23 \text { May } 2017 \\
\text { Available Online: } \\
10 \text { June } 2017\end{array}$ & $\begin{array}{l}\text { showed significantly lowest physiological loss in weight (PLW per cent), highest } \\
\text { fruit firmness }\left(\mathrm{Kg} \mathrm{cm}^{-1}\right) \text {, highest percentage of sound fruits (Per cent) and longest } \\
\text { shelf life (days) were recorded in the fruits stored under refrigerated condition } \\
\left(\mathrm{S}_{1}\right) \text {. }\end{array}$ \\
\hline
\end{tabular}

\section{Introduction}

The bitter gourd (Momordica charantia L.) is cultivated throughout India as a vegetable crop during the warm seasons of the year. The nutritive value is high in proteins, minerals and vitamins. It has immense medicinal properties due to the presence of beneficial phytochemicals which are known to have antibiotic, antimutagenic, antioxidant, antiviral, antidiabetic and immunity enhancing properties (Grover and Yadav, 2004). A compound known as momordicin and charantin present in the bitter gourd is used in the treatment of diabetes in reducing blood sugar level (Lotlikar et al., 1966). The perishable nature of bitter gourd is a definite risk to the traders.

Pre and postharvest technologies are employed to reduce the spoilage or postharvest losses and also to increase the storability of the produce. Good prepackaging, transport and storage are especially important for bitter gourd because of their perishability (Talukder et al., 2004). The postharvest loss of vegetables in developing countries is 20-50 per cent and 525 per cent in developed countries (Amiruzzaman, 2000). Packaging has a great 
significance in reducing wastage of fresh fruits and vegetables. Hence, experiments were conducted to study the influence of pre packaging materials and storage environment on shelf life of bitter gourd.

\section{Materials and Methods}

The laboratory experiment was conducted during 2014 at PG laboratory, Department of Horticulture, Agricultural College and Research Institute, TNAU, Madurai to standardize the suitable prepackaging material and storage environment on shelf life of Bitter gourd cultivars $\mathrm{C}_{1}-$ Roma, $\mathrm{C}_{2}-\mathrm{CO} 1, \mathrm{C}_{3}-$ MAHY 101, $\mathrm{C}_{4}$ - US 6214 and $\mathrm{C}_{5^{-}}$Palee. The experiment was laid out in Factorial Completely Randomized Design with six treatments in factor one, $\mathrm{T}_{1}$ - Perforated poly bag (200 gauge $+1 \%$ ventilation), $\mathrm{T}_{2}$.Un perforated poly bag (200 gauge), $\mathrm{T}_{3}-$ Wetted gunny bag, $\mathrm{T}_{4}-$ Wetted cloth bag, $\mathrm{T}_{5}-\mathrm{CFB}$ box ( $1 \%$ ventilation) and $\mathrm{T}_{6}-$ Control (without any packing) and two treatments in factor two, Refrigerated condition $\left(S_{1}\right)$ and Ambient condition $\left(\mathrm{S}_{2}\right)$. The data were analyzed statistically and interpreted.

\section{Results and Discussion}

Bitter gourd, because of its high moisture content is inherently more liable to deteriorate under tropical conditions. Like any other horticultural crop even after harvest they are biologically active and carryout transpiration, respiration, ripening and other biochemical changes, which deteriorate the quality of the produce.

All the three factors viz., different packaging materials (P), cultivars (C) and storage environment ( $S$ ) and their interaction effect differed significantly in physiological loss in weight of bitter gourd.

Among the different packaging materials $P_{1}$ (Perforated poly bag - 200 gauge with $1 \%$ ventilation) recorded the lowest physiological loss in weight of (10.38 per cent). The highest physiological weight was registered in $\mathrm{P}_{6}$ (Control - without any packing) with (21.96per cent). Among the different cultivars treatment $\mathrm{C}_{2}(\mathrm{CO} 1)$ registered the lowest physiological loss in weight of (13.14 per cent). The highest physiological loss in weight was registered in $\mathrm{C}_{4}$ (US 6214) (17.92 per cent). It could be due to, bitter gourds are packed with polyethylene bags records less moisture loss due to maintenance of high humidity surrounding bitter gourds, which in turn lower rate of transpiration. These results are in conformity with the results of Viraktamath et al., (1963) in brinjal and Adamicki, (1985) in cucumber. Among the different storage conditions S1 (Refrigerated condition) registered the lowest physiological loss of (7.74 per cent). The highest physiological loss was registered in $\mathrm{S}_{2}$ (Ambient condition) (23.59per cent). Modified atmosphere packaging is used in storage of fresh fruits and vegetables; the term refers to their storage in plastic films, which restrict the transmission of respiratory gases. This results in the accumulation of carbon dioxide and depletion of oxygen around the crop, which may increase the storage life (Kader et al., 1989).

The interaction effect of different packaging materials and cultivars $\mathrm{P}_{1} \mathrm{C}_{2}$ (Perforated poly bag - 200 gauge with $1 \%$ ventilation + CO1) registered the lowest physiological loss in weight of (8.49 per cent) and the highest physiological loss in weight was registered in $\mathrm{P}_{6} \mathrm{C}_{4}$ (Control - without any packing + US 6214) (23.34 per cent). The interaction effect of different cultivars and storage conditions $\mathrm{C}_{2} \mathrm{~S}_{1} \quad$ (CO1 +Refrigerated condition) registered the lowest physiological loss in weight of (6.92 percent) and the highest physiological loss was registered in $\mathrm{C}_{4} \mathrm{~S}_{2}$ (US 6214 + Ambient condition) (27.22 per cent). The interaction effect of different packaging 
materials and storage conditions $\mathrm{P}_{1} \mathrm{~S}_{1}$ (Perforated poly bag -200 gauge with $1 \%$ ventilation+ Refrigerated condition) registered the lowest physiological loss in weight of (4.78 per cent) and the highest physiological loss in weight was registered in $\mathrm{P}_{6} \mathrm{~S}_{2}$ (Control - without any packing + Ambient condition) (30.99 per cent). Patil et al., (2010) reported that, the physiological loss in weight during storage occurs continuously due to moisture loss; thereby the fruits lose their freshness. In the present study the transpiration and respiration could have caused loss of turgor in the fruits of bitter gourd. The rate of deterioration varied widely depending upon the commodity and storage conditions as observed earlier by various workers. In the case of bitter gourd, the fruits stored well up to 3-5 days (Sankaran, 1999). Jayaraman and Raju (1992) and Perkins Veazie and Collins (1992) observed similar results in bhendi.

Regarding the interaction effect of different packaging materials + cultivars + storage conditions $\mathrm{P}_{1} \mathrm{~S}_{1} \mathrm{C}_{2}$ (Perforated poly bag - 200 gauge with $1 \%$ ventilation + Refrigerated condition + CO1) registered the lowest physiological loss in weight of (4.28 per cent) and the highest physiological loss in weight was registered in $\mathrm{P}_{6} \mathrm{~S}_{2} \mathrm{C}_{4}$ (Control - without any packing + Ambient condition + US 6214) (32.30 per cent). The reduction in physiological loss in weight of gherkin stored in ventilated polythene bags arrest moisture loss and maintained turgidity. However, oxygen depletion, $\mathrm{CO}_{2}$ accumulation occurred in polythene bags resulting in low rate of respiration (Bindiya and Srihari, 2013). Similar results were observed by Attri et al., (2002) in chilli and Mangal et al., (2001) in brinjal. High $\mathrm{CO}_{2}$ can inhibit ethylene action as well as autocatalytic production of ethylene in climacteric fruits. Modified atmospheres can be defined as one that is created by altering the normal composition of air (78\% nitrogen, $21 \%$ oxygen, $0.03 \%$ carbon dioxide and traces of noble gases) to provide an optimum atmosphere for increasing the storage period and maintaining the quality of produce (Table 1).

Among the different packaging materials $\mathrm{P}_{1}$ (Perforated poly bag - 200 gauge with $1 \%$ ventilation) recorded the significantly highest fruit firmness of $4.88\left(\mathrm{~kg} \mathrm{~cm}^{-1}\right)$. The lowest fruit firmness was registered in $\mathrm{P}_{6}$ (Control without any Packing) with the value of $2.74\left(\mathrm{~kg} \mathrm{~cm}^{-1}\right)$. The different storage conditions $\mathrm{S}_{1}$ (Refrigerated condition) registered significantly highest fruit firmness of $4.54\left(\mathrm{~kg} \mathrm{~cm}^{-1}\right)$ and the lowest fruit firmness was registered in $S_{2}$ (Ambient condition) $3.41\left(\mathrm{~kg} \mathrm{~cm}^{-1}\right)$. The interaction effect of different packaging materials and cultivars $\mathrm{P}_{1} \mathrm{C}_{2}$ (Perforated poly bag - 200 gauge with 1 $\%$ ventilation $+\mathrm{CO} 1)$ registered significantly highest fruit firmness of $5.22\left(\mathrm{~kg} \mathrm{~cm}^{-1}\right)$ and the lowest fruit firmness was registered in $\mathrm{P}_{6} \mathrm{C}_{4}$ (Control - without any packing + US 6214) $2.15\left(\mathrm{~kg} \mathrm{~cm} \mathrm{~cm}^{-1}\right)$. The extended shelf life observed with ventilated bags may be due to optimum level of humidity and modified gaseous composition inside the bags which did not favour the growth of fungus, but in polyethylene bags without ventilation, which favours fungal growth leading to reduced storage life. These results are in confirmation with the results obtained by earlier workers Anandaswamy et al., (1989)in capsicum, Lingaiah et al., (1983) in bell pepper, Badgujar et al., (1987) and Viraktamath et al., (1963) in brinjal, Saimbhi and Ranohawa, (1983) in okra.

The interaction effect of different cultivars and storage conditions $\mathrm{C}_{2} \mathrm{~S}_{1} \quad(\mathrm{CO} 1$ + Refrigerated condition) registered the highest fruit firmness of $4.94\left(\mathrm{~kg} \mathrm{~cm}^{-1}\right)$ and the lowest fruit firmness was registered in $\mathrm{C}_{4} \mathrm{~S}_{2}$ (US $6214+$ Ambient condition) $3.03(\mathrm{~kg}$ $\mathrm{cm}^{-1}$ ). The interaction effect of different 
packaging materials and storage conditions $\mathrm{P}_{1} \mathrm{~S}_{1}$ (Perforated poly bag -200 gauge with 1 $\%$ ventilation+ Refrigerated condition) registered significantly highest fruit firmness of $5.75\left(\mathrm{~kg} \mathrm{~cm}^{-1}\right)$. The lowest fruit firmness was registered in $\mathrm{P}_{6} \mathrm{~S}_{2}$ (Control - without any packing + Ambient condition) $2.55\left(\mathrm{~kg} \mathrm{~cm}^{-1}\right)$. The firmness of bitter gourd fruits in terms of pressure was found to be reduced with the increase in the storage period. However, fruits packed with polyethylene bags with ventilation were more firm than control. This can be attributed mainly due to more loss of moisture from the control fruits. Where packaging helped to prevent moisture stress and softening and thereby maintained a high firmness. Which were obtained by various researchers Yehoshua et al., (1979) in tomato and Yehoshua et al., (1983), Miller et al., (1986) in bell pepper and Showalter, (1973) in green capsicum.

Among the interaction effect of different packaging materials + cultivars + storage conditions $\mathrm{P}_{1} \mathrm{~S}_{1} \mathrm{C}_{2}$ (Perforated poly bag - 200 gauge with $1 \%$ ventilation + Refrigerated condition $+\mathrm{CO} 1)$ registered the significantly highest fruit firmness $6.11\left(\mathrm{~kg} \mathrm{~cm}^{-1}\right)$ and the lowest fruit firmness was registered in $\mathrm{P}_{6} \mathrm{~S}_{2} \mathrm{C}_{4}$ (Control - without any packing + Ambient condition + US 6214) $2.05\left(\mathrm{~kg} \mathrm{~cm}^{-1}\right)$ (Table 2).

Among the different packaging materials $\mathrm{P}_{1}$ (Perforated poly bag - 200 gauge with $1 \%$ ventilation) recorded significantly highest sound fruits percentage of 83.59 per cent. The lowest percentage of sound fruits was registered in $\mathrm{P}_{6}$ (Control - without any packing) 71.96per cent (Table 3).

The different cultivars treatment $\mathrm{C}_{2}$ (CO1) registered the highest sound fruits percentage of 80.79 per cent and the lowest percentage of sound fruits was registered in $\mathrm{C}_{4}$ (US 6214) with the value of 76.02 per cent. Among the different storage conditions $\mathrm{S}_{1}$ (Refrigerated condition) registered significantly highest sound fruits of 86.22per cent and the lowest percentage of sound fruits was registered in $S_{2}$ (Ambient condition) (70.29 per cent). The highest sound fruit leads to delayed ripening by effect of reduced ethylene concentration and modified atmospheric condition of low $\mathrm{O}_{2}$ and enhanced $\mathrm{CO}_{2}$ in packaging. Similar findings were also reported by Waskar et al., (1999), Patil et al., (2010) in bottle gourd and Elangovan et al., (2006) in tomato.

Among the interaction effect of different packaging materials and cultivars $\mathrm{P}_{1} \quad \mathrm{C}_{2}$ (Perforated poly bag - 200 gauge with $1 \%$ ventilation $+\mathrm{CO} 1)$ registered significantly highest sound fruits of 85.51 per cent and the lowest percentage was registered in $\mathrm{P}_{6} \mathrm{C}_{4}$ (Control - without any packing + US 6214) (70.61 per cent). The interaction effect of different packaging materials and storage conditions $\mathrm{P}_{1} \mathrm{~S}_{1}$ (Perforated poly bag -200 gauge with $1 \%$ ventilation + Refrigerated storage) registered significantly highest sound fruits of 89.13 per cent and the lowest percentage of sound fruits was registered in $\mathrm{P}_{6} \mathrm{~S}_{2}$ (Control - without any packing + Ambient condition) (62.92 per cent). Regarding the interaction effect of different packaging materials + cultivars + storage conditions $\mathrm{P}_{1} \mathrm{~S}_{1} \mathrm{C}_{2}$ (Perforated poly bag - 200 gauge with $1 \%$ ventilation + Refrigerated condition $+\mathrm{CO}_{1}$ ) registered the significantly highest sound fruits percentage of 89.69 per cent. The lowest percentage of sound fruits was registered in $\mathrm{P}_{6} \mathrm{~S}_{2} \mathrm{C}_{4}$ (Control - without any packing + Ambient condition + US 6214) (61.54 per cent). The modified atmosphere packaging is used in storage of fresh fruits and vegetables; the term refers to their storage in plastic films, which restrict the transmission of respiratory gases. This results in accumulation of carbon dioxide and depletion of oxygen around the crop, which may increase their storage life (Kader et al., 1989). The different packaging materials $P_{1}$ 
(Perforated poly bag - 200 gauge with $1 \%$ ventilation) recorded significantly highest shelf life 5.44 days and the lowest shelf life was registered in $\mathrm{P}_{6}$ (Control - without any packing) (4.26 days).

Table.1 Effect of prepackaging materials and storage condition on physiological loss in weight (per cent) of bitter gourd cultivars

\begin{tabular}{|c|c|c|c|c|c|c|}
\hline TREATMENTS & $\mathrm{C}_{1}$ & $\mathrm{C}_{2}$ & $\mathrm{C}_{3}$ & $\mathrm{C}_{4}$ & $\mathrm{C}_{5}$ & MEAN \\
\hline $\mathbf{P}_{1}$ & 11.05 & 8.49 & 11.47 & 12.08 & 8.80 & 10.38 \\
\hline $\mathbf{P}_{2}$ & 19.44 & 18.03 & 19.46 & 20.23 & 18.56 & 19.14 \\
\hline $\mathbf{P}_{3}$ & 15.48 & 10.75 & 15.79 & 16.90 & 11.30 & 14.04 \\
\hline $\mathbf{P}_{4}$ & 16.42 & 10.82 & 17.15 & 17.74 & 11.64 & 14.75 \\
\hline $\mathbf{P}_{5}$ & 14.98 & 10.33 & 15.38 & 17.22 & 10.65 & 13.71 \\
\hline $\mathbf{P}_{6}$ & 21.83 & 20.40 & 22.54 & 23.34 & 21.70 & 21.96 \\
\hline MEAN & 16.53 & 13.14 & 16.96 & 17.92 & 13.77 & 15.66 \\
\hline $\mathbf{S}_{1}$ & 7.76 & 6.92 & 8.02 & 8.62 & 7.38 & 7.74 \\
\hline $\mathbf{S}_{2}$ & 25.30 & 19.35 & 25.90 & 27.22 & 20.17 & 23.59 \\
\hline MEAN & 16.53 & 13.14 & 16.96 & 17.92 & 13.77 & 15.66 \\
\hline $\mathbf{P}_{1} \mathbf{S}_{1}$ & 4.79 & 4.28 & 5.18 & 5.24 & 4.40 & 4.78 \\
\hline $\mathbf{P}_{1} \mathbf{S}_{2}$ & 17.30 & 12.70 & 17.76 & 18.92 & 13.20 & 15.98 \\
\hline $\mathbf{P}_{2} \mathbf{S}_{1}$ & 9.14 & 8.10 & 9.20 & 9.71 & 8.60 & 8.95 \\
\hline $\mathbf{P}_{2} \mathbf{S}_{2}$ & 29.73 & 27.96 & 29.71 & 30.74 & 28.51 & 29.33 \\
\hline $\mathbf{P}_{3} \mathbf{S}_{1}$ & 6.60 & 5.80 & 6.75 & 7.20 & 6.27 & 6.52 \\
\hline $\mathbf{P}_{3} \mathbf{S}_{2}$ & 24.35 & 15.70 & 24.83 & 26.60 & 16.32 & 21.56 \\
\hline $\mathbf{P}_{4} \mathbf{S}_{1}$ & 6.90 & 6.23 & 7.31 & 7.62 & 6.50 & 6.91 \\
\hline $\mathbf{P}_{\mathbf{4}} \mathbf{S}_{2}$ & 25.94 & 15.41 & 26.99 & 27.85 & 16.78 & 22.59 \\
\hline $\mathbf{P}_{5} \mathbf{S}_{1}$ & 6.24 & 5.71 & 6.29 & 7.55 & 5.90 & 6.34 \\
\hline $\mathbf{P}_{5} \mathbf{S}_{2}$ & 23.71 & 14.95 & 24.46 & 26.89 & 15.40 & 21.08 \\
\hline $\mathbf{P}_{6} \mathbf{S}_{1}$ & 12.87 & 11.40 & 13.41 & 14.38 & 12.58 & 12.93 \\
\hline $\mathbf{P}_{6} \mathbf{S}_{2}$ & 30.78 & 29.40 & 31.67 & 32.30 & 30.81 & 30.99 \\
\hline MEAN & 16.53 & 13.14 & 16.96 & $\mathbf{1 7 . 9 2}$ & 13.77 & 15.66 \\
\hline SOURCE & \multicolumn{3}{|c|}{ SEd } & \multicolumn{3}{|c|}{ CD $(P=0.05)$} \\
\hline $\mathbf{P}$ & \multicolumn{3}{|c|}{0.125} & \multicolumn{3}{|c|}{0.247} \\
\hline $\mathbf{S}$ & \multicolumn{3}{|c|}{0.072} & \multicolumn{3}{|c|}{0.142} \\
\hline $\mathrm{C}$ & \multicolumn{3}{|c|}{0.114} & \multicolumn{3}{|c|}{0.226} \\
\hline $\mathbf{P} \times S$ & \multicolumn{3}{|c|}{0.176} & \multicolumn{3}{|c|}{0.350} \\
\hline$S \times C$ & \multicolumn{3}{|c|}{0.161} & \multicolumn{3}{|c|}{0.319} \\
\hline$P \times C$ & \multicolumn{3}{|c|}{0.279} & \multicolumn{3}{|c|}{0.553} \\
\hline$P \times S \times C$ & \multicolumn{3}{|c|}{0.395} & \multicolumn{3}{|c|}{0.782} \\
\hline
\end{tabular}


Table.2 Effect of prepackaging materials and storage condition on fruit firmness $\left(\mathrm{kg} \mathrm{cm}^{-1}\right)$ of bitter gourd cultivars

\begin{tabular}{|c|c|c|c|c|c|c|}
\hline TREATMENTS & $\mathrm{C}_{1}$ & $\mathrm{C}_{2}$ & $\mathrm{C}_{3}$ & $\mathrm{C}_{4}$ & $\mathrm{C}_{5}$ & MEAN \\
\hline $\mathbf{P}_{1}$ & 4.90 & 5.22 & 4.76 & 4.50 & 5.02 & 4.88 \\
\hline $\mathbf{P}_{2}$ & 3.50 & 3.74 & 3.29 & 3.03 & 3.68 & 3.45 \\
\hline $\mathbf{P}_{3}$ & 4.13 & 4.41 & 3.90 & 3.72 & 4.24 & 4.08 \\
\hline $\mathbf{P}_{4}$ & 3.93 & 4.21 & 3.78 & 3.55 & 4.02 & 3.90 \\
\hline $\mathbf{P}_{5}$ & 4.81 & 5.06 & 4.68 & 4.41 & 4.98 & 4.79 \\
\hline $\mathbf{P}_{6}$ & 2.70 & 3.26 & 2.51 & 2.15 & 3.11 & 2.74 \\
\hline MEAN & 3.99 & 4.32 & 3.82 & 3.56 & 4.17 & 3.97 \\
\hline $\mathbf{S}_{1}$ & 4.52 & 4.94 & 4.40 & 4.10 & 4.74 & 4.54 \\
\hline $\mathbf{S}_{2}$ & 3.47 & 3.69 & 3.24 & 3.02 & 3.61 & 3.41 \\
\hline MEAN & 3.99 & 4.32 & 3.82 & 3.56 & 4.17 & 3.97 \\
\hline $\mathbf{P}_{1} \mathbf{S}_{1}$ & 5.80 & 6.11 & 5.67 & 5.32 & 5.87 & 5.75 \\
\hline $\mathbf{P}_{1} \mathbf{S}_{2}$ & 4.00 & 4.33 & 3.85 & 3.67 & 4.17 & 4.00 \\
\hline $\mathbf{P}_{2} \mathbf{S}_{1}$ & 3.80 & 4.16 & 3.66 & 3.41 & 3.99 & 3.80 \\
\hline $\mathbf{P}_{2} \mathbf{S}_{2}$ & 3.20 & 3.31 & 2.92 & 2.65 & 3.36 & 3.09 \\
\hline $\mathbf{P}_{3} \mathbf{S}_{1}$ & 4.66 & 4.96 & 4.50 & 4.23 & 4.68 & 4.61 \\
\hline $\mathbf{P}_{3} \mathbf{S}_{2}$ & 3.60 & 3.86 & 3.30 & 3.21 & 3.80 & 3.55 \\
\hline $\mathbf{P}_{4} \mathbf{S}_{1}$ & 4.43 & 4.83 & 4.35 & 4.04 & 4.52 & 4.43 \\
\hline $\mathbf{P}_{4} \mathbf{S}_{2}$ & 3.43 & 3.58 & 3.21 & 3.06 & 3.52 & 3.36 \\
\hline $\mathbf{P}_{5} \mathbf{S}_{1}$ & 5.71 & 5.97 & 5.57 & 5.36 & 5.85 & 5.69 \\
\hline $\mathbf{P}_{5} \mathbf{S}_{2}$ & 3.90 & 4.15 & 3.79 & 3.46 & 4.10 & 3.88 \\
\hline $\mathbf{P}_{6} \mathbf{S}_{1}$ & 2.70 & 3.60 & 2.63 & 2.24 & 3.51 & 2.94 \\
\hline $\mathbf{P}_{6} \mathbf{S}_{2}$ & 2.70 & 2.92 & 2.38 & 2.05 & 2.70 & 2.55 \\
\hline MEAN & 3.99 & 4.32 & 3.82 & 3.56 & 4.17 & 3.97 \\
\hline SOURCE & \multicolumn{3}{|c|}{ SEd } & \multicolumn{3}{|c|}{ CD $(P=0.05)$} \\
\hline $\mathbf{P}$ & \multicolumn{3}{|c|}{0.025} & \multicolumn{3}{|c|}{0.050} \\
\hline $\mathbf{S}$ & \multicolumn{3}{|c|}{0.014} & \multicolumn{3}{|c|}{0.029} \\
\hline $\mathrm{C}$ & \multicolumn{3}{|c|}{0.023} & \multicolumn{3}{|c|}{0.046} \\
\hline $\mathbf{P} \times S$ & \multicolumn{3}{|c|}{0.036} & \multicolumn{3}{|c|}{0.071} \\
\hline $\mathbf{S} \times \mathbf{C}$ & \multicolumn{3}{|c|}{0.032} & \multicolumn{3}{|c|}{0.065} \\
\hline $\mathbf{P} \times \mathbf{C}$ & \multicolumn{3}{|c|}{0.056} & \multicolumn{3}{|c|}{0.112} \\
\hline$P \times S \times C$ & \multicolumn{3}{|c|}{0.080} & \multicolumn{3}{|c|}{0.159} \\
\hline
\end{tabular}


Table.3 Effect of prepackaging materials and storage condition on sound fruits (per cent) of bitter gourd cultivars

\begin{tabular}{|c|c|c|c|c|c|c|}
\hline TREATMENTS & $\mathrm{C}_{1}$ & $\mathrm{C}_{2}$ & $\mathrm{C}_{3}$ & $\mathrm{C}_{4}$ & $\mathrm{C}_{5}$ & MEAN \\
\hline $\mathbf{P}_{1}$ & 82.97 & 85.51 & 82.67 & 81.71 & 85.12 & 83.59 \\
\hline $\mathbf{P}_{2}$ & 74.44 & 76.87 & 74.47 & 73.73 & 75.94 & 75.09 \\
\hline $\mathbf{P}_{3}$ & 78.59 & 82.85 & 78.42 & 77.10 & 82.10 & 79.81 \\
\hline $\mathbf{P}_{4}$ & 77.03 & 82.59 & 76.68 & 76.17 & 81.99 & 78.89 \\
\hline $\mathbf{P}_{5}$ & 79.11 & 83.51 & 78.51 & 76.83 & 83.12 & 80.22 \\
\hline $\mathbf{P}_{6}$ & 72.05 & 73.42 & 71.30 & 70.61 & 72.42 & 71.96 \\
\hline MEAN & 77.36 & 80.79 & 77.01 & 76.02 & 80.11 & 78.26 \\
\hline $\mathbf{S}_{1}$ & 86.27 & 87.06 & 85.95 & 85.31 & 86.53 & 86.22 \\
\hline $\mathbf{S}_{\mathbf{2}}$ & 68.46 & 74.52 & 68.07 & 66.74 & 73.69 & 70.29 \\
\hline MEAN & 77.36 & 80.79 & 77.01 & 76.02 & 80.11 & 78.26 \\
\hline $\mathbf{P}_{1} \mathbf{S}_{1}$ & 89.30 & 89.69 & 88.94 & 88.17 & 89.54 & 89.13 \\
\hline $\mathbf{P}_{1} \mathbf{S}_{2}$ & 76.64 & 81.32 & 76.39 & 75.25 & 80.70 & 78.06 \\
\hline $\mathbf{P}_{2} \mathbf{S}_{1}$ & 84.94 & 85.87 & 84.76 & 84.31 & 85.29 & 85.03 \\
\hline $\mathbf{P}_{2} \mathbf{S}_{2}$ & 63.94 & 67.86 & 64.18 & 63.15 & 66.58 & 65.14 \\
\hline $\mathbf{P}_{3} \mathbf{S}_{1}$ & 87.49 & 88.11 & 87.27 & 86.86 & 87.58 & 87.46 \\
\hline $\mathbf{P}_{3} \mathbf{S}_{2}$ & 69.69 & 77.58 & 69.56 & 67.33 & 76.61 & 72.15 \\
\hline $\mathbf{P}_{4} \mathbf{S}_{1}$ & 87.02 & 87.90 & 86.56 & 86.44 & 87.41 & 87.07 \\
\hline $\mathbf{P}_{4} \mathbf{S}_{2}$ & 67.04 & 77.27 & 66.80 & 65.89 & 76.57 & 70.71 \\
\hline $\mathbf{P}_{5} \mathbf{S}_{1}$ & 87.87 & 88.32 & 87.62 & 86.39 & 88.08 & 87.66 \\
\hline $\mathbf{P}_{5} \mathbf{S}_{2}$ & 70.34 & 78.70 & 69.40 & 67.27 & 78.16 & 72.77 \\
\hline $\mathbf{P}_{6} \mathbf{S}_{1}$ & 81.01 & 82.48 & 80.53 & 79.68 & 81.30 & 81.00 \\
\hline $\mathbf{P}_{6} \mathbf{S}_{2}$ & 63.08 & 64.36 & 62.07 & 61.54 & 63.53 & 62.92 \\
\hline MEAN & 77.36 & 80.79 & 77.01 & 76.02 & 80.11 & 78.26 \\
\hline SOURCE & \multicolumn{3}{|c|}{ SEd } & \multicolumn{3}{|c|}{$\mathrm{CD}(\mathrm{P}=\mathbf{0 . 0 5})$} \\
\hline $\mathbf{P}$ & \multicolumn{3}{|c|}{0.542} & \multicolumn{3}{|c|}{1.073} \\
\hline $\mathbf{S}$ & \multicolumn{3}{|c|}{0.313} & \multicolumn{3}{|c|}{0.619} \\
\hline C & \multicolumn{3}{|c|}{0.495} & \multicolumn{3}{|c|}{0.980} \\
\hline$P \times S$ & \multicolumn{3}{|c|}{0.766} & \multicolumn{3}{|c|}{1.518} \\
\hline$S \times C$ & \multicolumn{3}{|c|}{0.700} & \multicolumn{3}{|c|}{1.386} \\
\hline $\mathbf{P} \times \mathbf{C}$ & \multicolumn{3}{|c|}{1.212} & \multicolumn{3}{|c|}{ NS } \\
\hline$P \times S \times C$ & \multicolumn{3}{|c|}{1.714} & \multicolumn{3}{|c|}{ NS } \\
\hline
\end{tabular}


Table.4 Effect of prepackaging materials and storage condition on shelf life (days) of bitter gourd cultivars

\begin{tabular}{|c|c|c|c|c|c|c|}
\hline TREATMENTS & $\mathrm{C}_{1}$ & $\mathrm{C}_{2}$ & $\mathbf{C}_{3}$ & $\mathrm{C}_{4}$ & $\mathrm{C}_{5}$ & MEAN \\
\hline $\mathbf{P}_{1}$ & 5.29 & 5.95 & 5.16 & 5.01 & 5.81 & 5.44 \\
\hline $\mathbf{P}_{2}$ & 4.55 & 4.76 & 4.45 & 4.39 & 4.70 & 4.57 \\
\hline $\mathbf{P}_{3}$ & 4.96 & 5.13 & 4.92 & 4.78 & 5.05 & 4.97 \\
\hline $\mathbf{P}_{4}$ & 4.79 & 4.90 & 4.67 & 3.54 & 4.88 & 4.55 \\
\hline $\mathbf{P}_{5}$ & 5.19 & 5.70 & 5.07 & 4.97 & 5.65 & 5.31 \\
\hline $\mathbf{P}_{6}$ & 4.23 & 4.53 & 4.14 & 4.00 & 4.40 & 4.26 \\
\hline MEAN & 4.83 & 5.16 & 4.73 & 4.45 & 5.08 & 4.85 \\
\hline $\mathbf{S}_{1}$ & 5.74 & 6.23 & 5.64 & 5.13 & 6.14 & 5.78 \\
\hline $\mathbf{S}_{\mathbf{2}}$ & 3.92 & 4.09 & 3.83 & 3.76 & 4.02 & 3.92 \\
\hline MEAN & 4.83 & 5.16 & 4.73 & 4.45 & 5.08 & 4.85 \\
\hline $\mathbf{P}_{1} \mathbf{S}_{1}$ & 6.35 & 7.49 & 6.21 & 6.00 & 7.31 & 6.67 \\
\hline $\mathbf{P}_{1} \mathbf{S}_{2}$ & 4.23 & 4.40 & 4.10 & 4.02 & 4.31 & 4.21 \\
\hline $\mathbf{P}_{2} \mathbf{S}_{1}$ & 5.42 & 5.61 & 5.36 & 5.28 & 5.55 & 5.44 \\
\hline $\mathbf{P}_{2} \mathbf{S}_{2}$ & 3.67 & 3.90 & 3.54 & 3.49 & 3.84 & 3.69 \\
\hline $\mathbf{P}_{3} \mathbf{S}_{1}$ & 5.92 & 6.12 & 5.84 & 5.61 & 6.00 & 5.90 \\
\hline $\mathbf{P}_{3} \mathbf{S}_{2}$ & 4.00 & 4.13 & 4.00 & 3.95 & 4.09 & 4.03 \\
\hline $\mathbf{P}_{4} \mathbf{S}_{1}$ & 5.65 & 5.80 & 5.47 & 3.34 & 5.78 & 5.21 \\
\hline $\mathbf{P}_{4} \mathbf{S}_{2}$ & 3.92 & 4.00 & 3.87 & 3.74 & 3.97 & 3.90 \\
\hline $\mathbf{P}_{5} \mathbf{S}_{1}$ & 6.21 & 7.14 & 6.10 & 5.93 & 7.10 & 6.50 \\
\hline $\mathbf{P}_{5} \mathbf{S}_{2}$ & 4.16 & 4.26 & 4.04 & 4.00 & 4.20 & 4.13 \\
\hline $\mathbf{P}_{6} \mathbf{S}_{1}$ & 4.91 & 5.20 & 4.86 & 4.61 & 5.09 & 4.93 \\
\hline $\mathbf{P}_{6} \mathbf{S}_{2}$ & 3.54 & 3.86 & 3.42 & 3.38 & 3.71 & 3.58 \\
\hline MEAN & 4.83 & 5.16 & 4.73 & 4.45 & 5.08 & 4.85 \\
\hline SOURCE & \multicolumn{3}{|c|}{ SEd } & \multicolumn{3}{|c|}{ CD $(P=0.05)$} \\
\hline $\mathbf{P}$ & \multicolumn{3}{|c|}{0.036} & \multicolumn{3}{|c|}{0.072} \\
\hline $\mathbf{S}$ & \multicolumn{3}{|c|}{0.021} & \multicolumn{3}{|c|}{0.041} \\
\hline $\mathrm{C}$ & \multicolumn{3}{|c|}{0.033} & \multicolumn{3}{|c|}{0.066} \\
\hline $\mathbf{P} \times S$ & \multicolumn{3}{|c|}{0.051} & \multicolumn{3}{|c|}{0.102} \\
\hline S x C & \multicolumn{3}{|c|}{0.047} & \multicolumn{3}{|c|}{0.093} \\
\hline $\mathbf{P} \times \mathbf{C}$ & \multicolumn{3}{|c|}{0.081} & \multicolumn{3}{|c|}{0.161} \\
\hline$P \times S \times C$ & \multicolumn{3}{|c|}{0.115} & \multicolumn{3}{|c|}{0.228} \\
\hline
\end{tabular}


Fig.1 Packaging materials used for the study

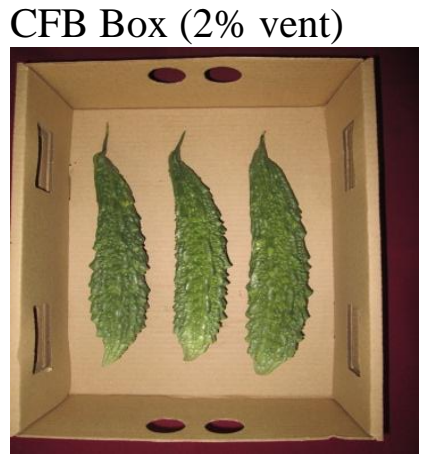

Un perforated Poly bag (200 gauge)

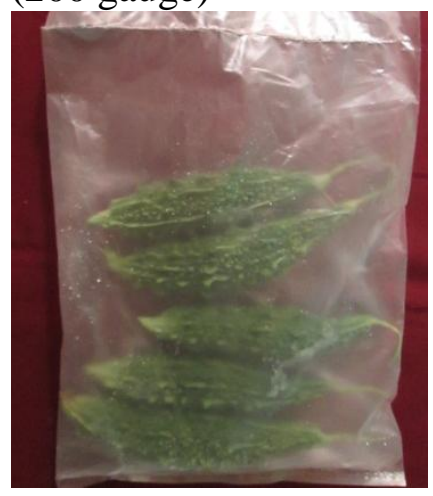

Wetted Gunny bag

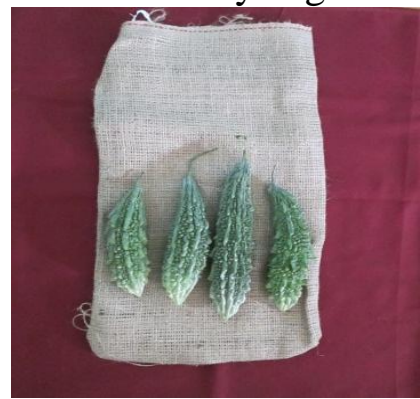

Perforated poly bag (200 gauge $+1 \%$ ventilation)

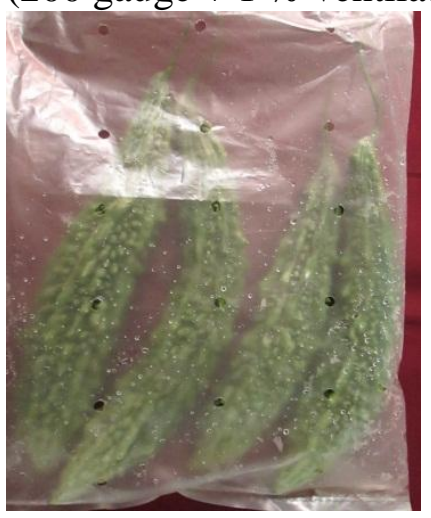

Wetted cloth bag

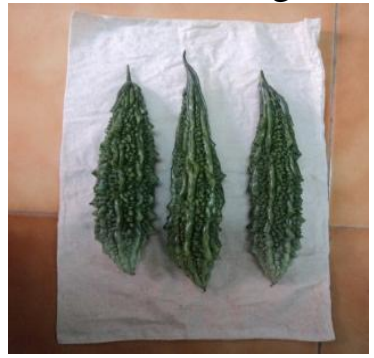

Cultivars treatment $\mathrm{C}_{2}(\mathrm{CO} 1)$ registered the highest shelf life of 5.16 days and the lowest shelf life was registered in $\mathrm{C}_{4}$ (US 6214) (4.45 days). Among the different storage conditions $\mathrm{S}_{1}$ (Refrigerated condition) registered the significantly highest shelf life of 5.78 days. The lowest shelf life was registered in S2 (Ambient condition) (3.92 days). The shelf life of fruits and vegetables is also enhanced at low temperature or under cold storage conditions (Dalal and Subramanyam, 1970). Roy and Khurdiya, (1983) have designed zero energy cool chambers which are reported to enhance the shelf life of vegetables by lowering the temperature and increasing the humidity inside the chambers.

The interaction effect of different packaging materials and cultivars $\mathrm{P}_{1} \mathrm{C}_{2}$ (Perforated poly bag - 200 gauge with $1 \%$ ventilation $+\mathrm{CO} 1)$ registered significantly highest shelf life of 5.95 days and the lowest shelf life was registered in $\mathrm{P}_{6} \mathrm{C}_{4}$ (Control - without any packing + US 6214) 4.00 days. Among the interaction effect of different cultivars and storage conditions $\mathrm{C}_{2} \mathrm{~S}_{1}$ (CO1 +Refrigerated condition) registered the highest shelf life of 6.23 days and the lowest shelf life was registered in $\mathrm{C}_{4} \mathrm{~S}_{2}$ (US $6214+$ Ambient condition) 3.76 days.

The interaction effect of different packaging materials and storage conditions $\mathrm{P}_{1} \mathrm{~S}_{1}$ (Perforated poly bag -200 gauge with $1 \%$ ventilation+ Refrigerated condition) registered significantly highest shelf life of 6.67 days and the lowest shelf life was registered in $\mathrm{P}_{6} \mathrm{~S}_{2}$ (Control - without any packing + Ambient condition) 3.58 days. Temperature plays a key role in the metabolism of fruits and vegetables (Marangoni et al., 1996). Number of chemical and physical processes takes place in vegetables during storage shelf life. 
Among the interaction effect of different packaging materials + cultivars + storage conditions $\mathrm{P}_{1} \mathrm{~S}_{1} \mathrm{C}_{2}$ (Perforated poly bag - 200 gauge with $1 \%$ ventilation + Refrigerated condition $+\mathrm{CO} 1)$ registered significantly highest shelf life of 7.49 days and the lowest shelf life was registered in $\mathrm{P}_{6} \mathrm{~S}_{2} \mathrm{C}_{4}$ (Control without any packing + Ambient condition + US 6214) 3.38 days. Fruits stored in polythene bags recorded lower spoilage than other packaging materials. This might be due to the high permeability of gases through the film to prevent anaerobic respiration (Chadha, 2001). The highest shelf life was recorded in fruits packed in polythene and stored in refrigerated condition, spoilage was delayed and shelf life of bitter gourds was extended. These results were in conformity with Glahan, (2009) in cabbage and Bindiya and Srihari, (2013) in gherkin (Table 4).

In conclusion, the study results indicated that perforated poly bag (200 gauge with $1 \%$ ventilation) plays a very effective role in controlling physiological loss in weight (PLW), Fruit firmness, Sound fruits per cent and Shelf life (days) of bitter gourd fruit stored under refrigerated condition. This may be due to the combination effect of prepackaging materials and storage environment.

\section{References}

Adamicki, F, 1985. Effect of storage temperature and wrapping on the keeping quality of cucumber fruits. ActaHorticulture., 156: 269-272.

Amiruzzaman, M. 2000. Postharvest technology / management (vegetables). A consultancy report., AVRDC- USAID. Bangaladesh project.Pp.193.

Anandaswamy, B., H. B. Murthy and N. V. R. Iyengar. 1959. Prepackaging studies of fresh produce. Capsicum grossum Sonat and Capsicum accumulatum Fing. Industrial research., 18:274.

Attri, B. L., KishanSwaroop and R. P. Medhi.
2002. Effect of storage on postharvest life of different cultivars of chilli (Capsicum frutescence) under tropical conditions of Andaman and Nicobar Islands. Indian J. Hort., 59(2): 171-176.

Badgujar, C. D., K. E. Lawande and P. N. Kale. 1987. Polythene packaging for increasing shelf life in brinjal fruits. Cur Res Reporter. Mahatma PhuleAgril. Univ., 3(2):22-25.

Bindiya, Y and D. Srihari. 2013. Influence of polyethylene packaging on shelf life and quality of gherkin (Cucumis anguriaL.) under ambient condition. Karnataka J. Agric. Sci., 26 (4): 534- 538.

Chadha, K. L., 2001. Hand Book of Horticulture. Indian Council of Agricultural Research, New Delhi.

Dalal, V. B and H. Subramanyam. 1970. Refrigerated storage of fresh fruits and vegetables. Climate control. 3(3): 37-50.

Elangovan, R., V. K. Batra., S. K. Arora., J. Kumar and V.P Singh. 2006. Studies on the effect of storage condition on the shelf life of tomato. Haryana Journal of Plant Science., 35(1-2): 130-134.

Glahan, S. 2009. Influence of packaging materials on changing of $\mathrm{CO}_{2}: \mathrm{O}_{2}$ during the storage of fresh cut red cabbage. ActaHorticulture., 837: 271-278.

Grover, J. K and S.P.Yadav. 2004. Pharmacological actions and potential uses of Momordica charantia: a review. J. Ethnopharmacol., 93: 123-132.

Jayaraman, K. S and P. S. Raju. 1992. Development and evaluation of a permanganate based ethylene scrubber for extending the shelf life of fresh fruits and vegetables. J. Food. Sci. Tech., 29(2): 7783.

Kader, A. A., D. Zagory and E. L. Kervel. 1989. Modified atmosphere packaging of fruits and vegetables. Cri. Rev. Food Sci. Nut., 28: 1-30.

Lingaiah, H. B., A. G. Hudder., P. M. Gowda and V. Chikkasubbanna. 1983. The influence of pre cooling, waxing and prepackaging on shelf life and quality of bell pepper. Proceedings of National Seminar on Production Technology of Tomato and Chillies. TNAU. India., 157-159.

Lotlikar, M. M and M. R. RajaramaRao. 1966. 
Pharmacology of hypoglycemic principle isolated from the fruit of Momordica charantia Linn. Indian J Pharm., 28: 129132.

Mangal, J. L., J. Kumar., V. K. Batra and J. Singh. 2001. Effect of cultivars, packaging types and waxing on shelf life of brinjal (Solanum melongena L.). Veg. Sci., 28(1): 43-44.

Marangoni, A. G., T, Palma and D.W. Stanley. 1996. Membrane effect in postharvest physiology. Postharvest Biology and Technology., 7: 193-217.

Miller, W. R., L. A. Risse and R. E. MecDonald. 1986. Deterioration of individual wrapped and non-wrapped bell peppers during long term storage. Trop. Sci., 26: 1-8.

Patil, P. D., B. R. Parmer., P. P. Bhalerao. 2010. Effect of storage and packaging materials on shelf life of bottle gourd (Lagenaria siceraria L.) cv. Pusa Naveen. The Asian Journal of Horticulture., 5(1): 214-217.

Perkins Veazie, P. and J. K. Collins. 1992. Packaging and storage temperature differences in postharvest shelf life of okra. Hort. Tech., 2(3): 350-352.

Roy, S. K and D. S. Khurdiya.1983. Zero energy chamber for storage of horticultural produce. Indian Agricultural Research Institute, New Delhi.

Saimbhi, M. S and Ranohawa. 1983. Shelf life of okra (Abelmoschus esculentusL. Moench) as influenced by prepackaging in polyethylene bags. Indian Food Packer., 37:63.

Sankaran, M. 1999. Harvesting maturity and transit temperature for the export of bhendi (Abelmoschus esculentusL. Moench), bitter gourd (Momordica charantia L.) and Drumstick (Moringa olefera Lam.)M.Sc., (Hort.) Thesis, Tamil Nadu Agricultural University, Coimbatore.

Showalter, R. K. 1973. Factors affecting pepper firmness. Proc. Fla. St. Hort. Sec., 86: 230232.

Talukder, S., K. M. Khalequzzaman., M. N. A. Choudry., S. M. K. E. Khuda and M. D. MasudAlam. 2004. Pre packaging, storage losses and physiological changes of fresh bitter gourd as influenced by post harvest treatments. Journal of Biological Sciences., 4 (5): 613-615.

Viraktamath, C. S., B. Anandaswamy., K. R. S. Rao., B. N. Suryanarayana., N. V. R. Iyengar and H. C. Srivastava. 1963. Prepackaging studies of fresh produce. Food Science., 12: 236-331.

Waskar, D. P., B. B. Yadav and V. K. Garande. 1999. Influence of various packaging materials on storage behavior of bottle gourd under different storage conditions. Indian Journal of Agricultural Research., 33(2): 287-292.

Yehoshua, B. S., B. Shipiro and Z. Even-chen. 1983. Mode of action of individual seal packaging in high density polyethylene (HDPE) film in delaying deterioration of lemon and bell pepper fruits. Proc. Int. Soc. Citriculture. Japan., 2: 718-721.

Yehoshua, B. S., I. Kobller and B. Shapiro. 1979. Effect of seal-packaging of individual tomatoes on rate of fruit deterioration. Prelim. Rep. Volcani Center. BetDagan No. 779, pp.17.

\section{How to cite this article:}

Rajasekar Manivelu, Swaminathan Venkatesan and Hemalatha Ganapathyswami. 2017. Standardization of Prepackaging Materials and Storage Environment on Post Harvest Shelf Life of Bitter Gourd (Momordica charantia L.). Int.J.Curr.Microbiol.App.Sci. 6(6): 1886-1896. doi: https://doi.org/10.20546/ijcmas.2017.606.220 